\title{
Modelling of Reduced GB Transmission System in PSCAD/EMTDC
}

\author{
Ronak Rabbani \\ Brunel University \\ Ronak.Rabbani@brunel.ac.uk
}

\author{
Mohsen Mohammadi \\ Brunel University \\ Mohsen.Mohammadi@brunel.ac.uk
}

\author{
Shadi Khaleghi Kerahroudi \\ Brunel University \\ Shadi.Kerahroudi@nationalgrid.com
}

\author{
Ahmed F. Zobaa \\ Brunel University \\ Ahmed.Zobaa@brunel.ac.uk
}

\author{
Gareth A. Taylor \\ Brunel University \\ Gareth.Taylor@brunel.ac.uk
}

\begin{abstract}
Energy and environmental issues are two of the greatest challenges facing the world today. In response to energy needs and environmental concerns, renewable energy technologies are now considered the future technologies of choice. Renewable energy is produced from natural sources that are clean and free; however, it is widely accepted that renewable energy is not a solution without challenges. An example of this can be seen in the UK, where there is much interest amongst generation developers in the construction of new large-scale onshore and offshore wind farms, especially in Scotland. The stability of electric power systems is also an important issue. It is important to have full knowledge of the system and to be able to predict the behaviour under different situations is an important objective. As a result, several industrial-grade power system simulator tools have been developed in order to estimate the behaviour of the electric power system under certain conditions. This paper presents a reduced Great Britain (GB) system model for stability analysis using PSCAD/EMTDC. The reduced model is based upon a future GB transmission system model and, hence, contains different types and mix of generation, HVDC transmission lines and additional interconnection. The model is based on the reduced DIgSILENT PowerFactory model developed by National Grid.
\end{abstract}

Index Terms- System Modeling, Software Comparison, PSCAD/EMTDC, DIgSILENT.

\section{INTRODUCTION}

The UK government has made wind energy a key component in the commitment to reduce man-made greenhouse gas emission levels. In particular, the number of large offshore and onshore wind farms in the UK is expected to increase considerably [1]. The GB network consists of an onshore transmission network, covering England, Wales and Scotland, and an offshore transmission network. A large percentage of its installed generation capacity consists of nonrenewable sources, such as gas/CHP, coal and nuclear. The proportion of generation mix is expected to reverse towards the middle of the next decade due to development of several renewable generation plants and the closure of coal and oil plants which are either close to the end of their working life or unable to meet the emission targets.[2]

The installed capacity is predicted to increase up to $18-20$ GW by 2020, compared to the current installed capacity of 10 GW, including 3.6 GW offshore [1], [3]. There will be key operational challenges for GB transmission networks, with increased wind penetration expected in Scotland. It is, therefore, planned to reinforce the GB electrical power transmission system between 2013 and 2022 [4], through the use of many more HVDC links operating in parallel with existing AC transmission routes, and also controllable reactive power sources such as Static VAr Compensation (SVC) and Thyristor Controlled Series Compensation (TCSC).

These changes will make the future GB transmission network unique in many aspects. Large penetration of wind power with changing wind velocity, direction and location will shift the generation concentration from one part of the system to another. Consequently, power flow patterns in the network will experience large variation. In addition, the island network, with several HVDC interconnections with external grids, internal HVDC transmission lines and offshore HVDC networks, will give rise to a power system with reasonably high concentration of FACTS devices. These devices can offer various supplementary control features to improve the reliability and stability of the power system. However, they can cause new problems in the network [2]. Voltage stability is an important issue in an electrical power system. Consequently, several industrial-grade power system simulator tools have been developed in order to estimate the behaviour of the electric power system under certain conditions.

The system presented in this paper is a reduced GB system model. The reduced model is based upon a future GB transmission system model and, hence, contains different types and mix of generation. This model is also based on the reduced DIgSILENT PowerFactory model developed by National Grid. Also, the aim of this paper is to compare PSCAD with the more widespread simulator, DIgSILENT PowerFactory. The tools employ different models, components and analytical and numerical algorithms; therefore, different results can be expected for the same system.

Section II gives the introduction of PSCAD/EMTDC and DIgSILENT. The power system and component modelling are described in section III. Section IV provides the power flow results of the PSCAD/EMTDC program also a comparison between both software tools results and, finally, a conclusion is proposed in section VI. 


\section{INTRODUCTION OF PSCAD/EMTDC AND DIGSILENT}

These software packages are normally developed for a specific area of the power system to improve the analytical ability and computational efficiency [5]. The manufacturers of these software packages also effort to make them as user friendly as possible, especially those used in research and for educational purposes. PSCAD/EMTDC and DIgSILENT PowerFactory are two examples of these software packages [5].

\section{A. PSCAD/EMTDC}

The simulation of power system in time domain and frequency domain is the main function of PSCAD. It also can be used in harmonic research of AC system, analysis of transient torque, the HVDC system and HVDC commutation [5]. It can simulate for the electromagnetic transient process of a series or parallel multi-terminal transmission system for $\mathrm{AC} / \mathrm{DC}$ system, also the interaction between the parallel AC and DC lines on the same tower. EMTDC program has the "snapshot" function, which means the sections at some time instants of the system can be recorded. Based on this function further study on system transient process can be carried on [5].

The library of the PSCAD/EMTDC almost includes all kinds of elements in power system. This software is also capable to interface to MATLAB, through which we can

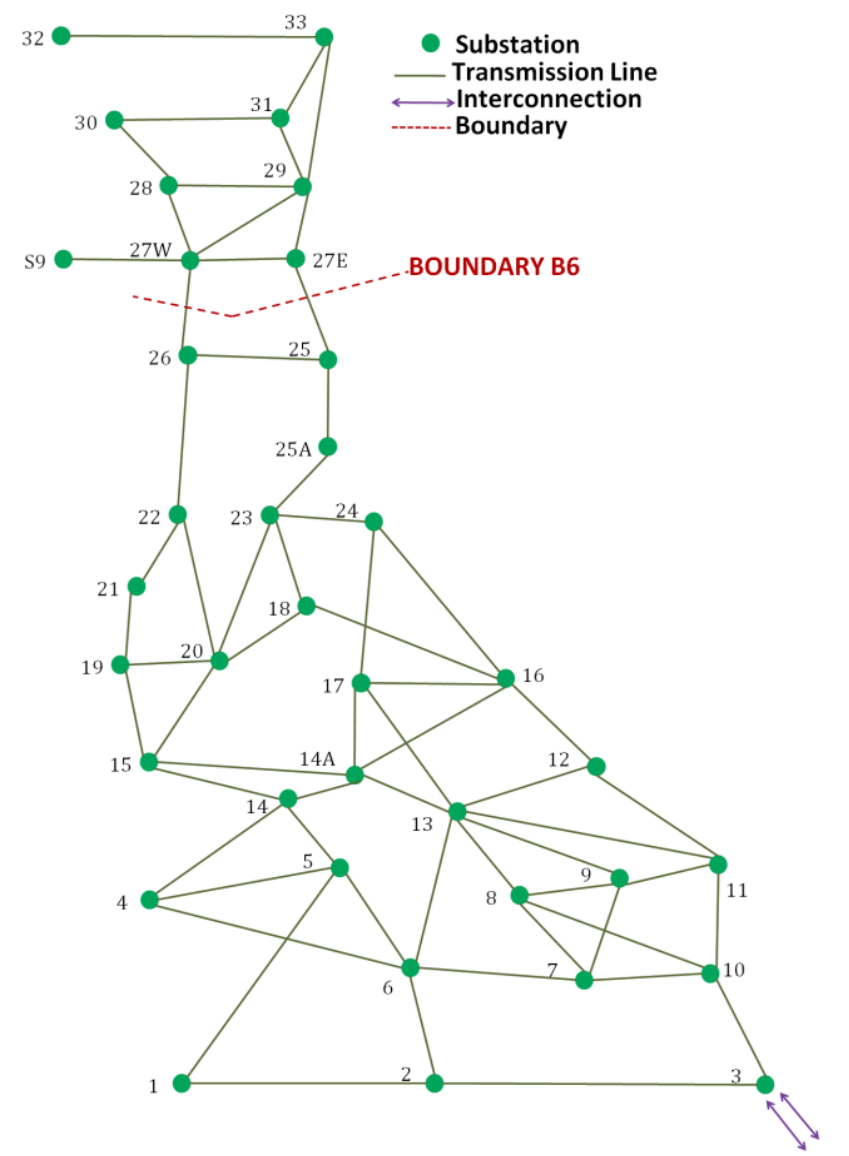

Fig. 1. Representative GB transmission network

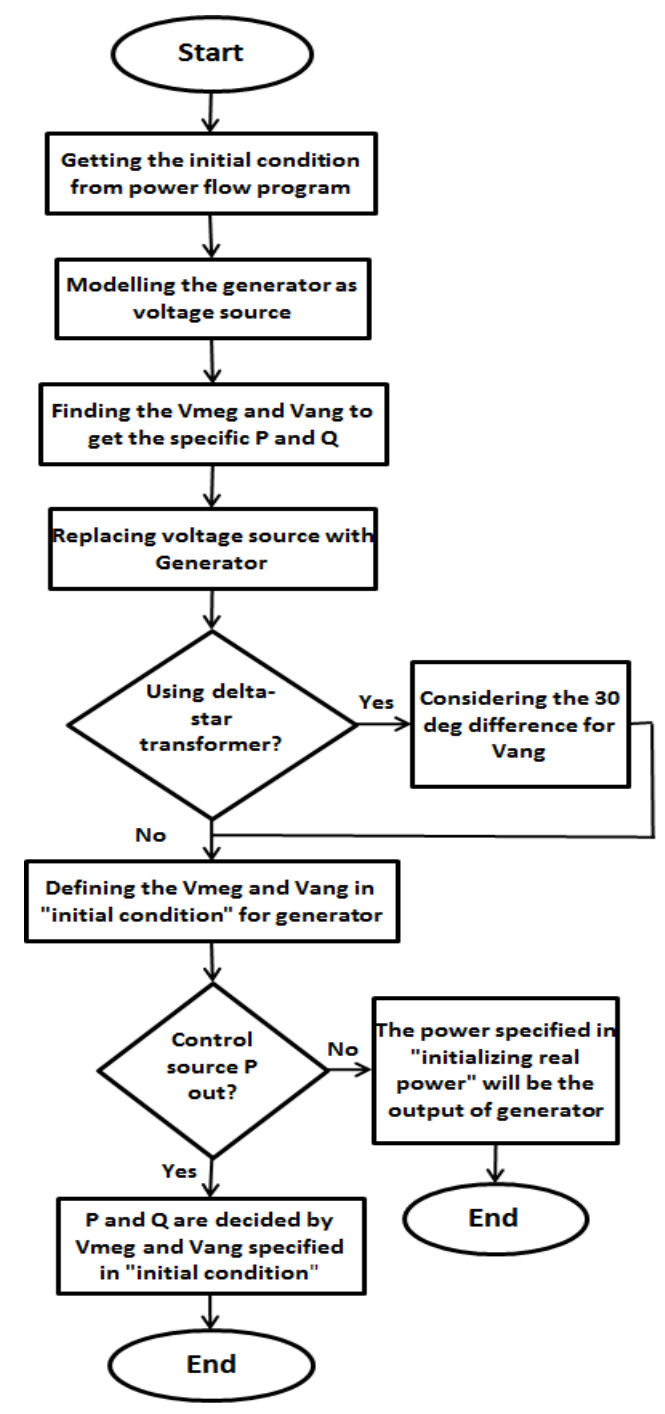

Fig. 2. Algorithm of Modelling the Generator in the PSCAD

easily use the visual numerical calculation function in MATLAB [6].

\section{B. DIgSILENT}

DIgSILENT PowerFactory is an integrated power analysis tool that combines reliable and flexible system modelling capabilities with state-of-the-art solution algorithms and unique object-oriented database management. The load-flow, short-circuit calculations, harmonic analysis, protection coordination; stability calculation and modal analysis have been included in this software package [7].

\section{Methodology}

\section{A. Power System Modelling}

Fig. 1 shows a single-line diagram of the model consisting of 37 substations, inter-connected through 64 transmission lines. It contains 67 generators of various generation types, 23 SVCs and 36 loads. These network branches are intended to represent the main routes on which power flows across the GB transmission system. 


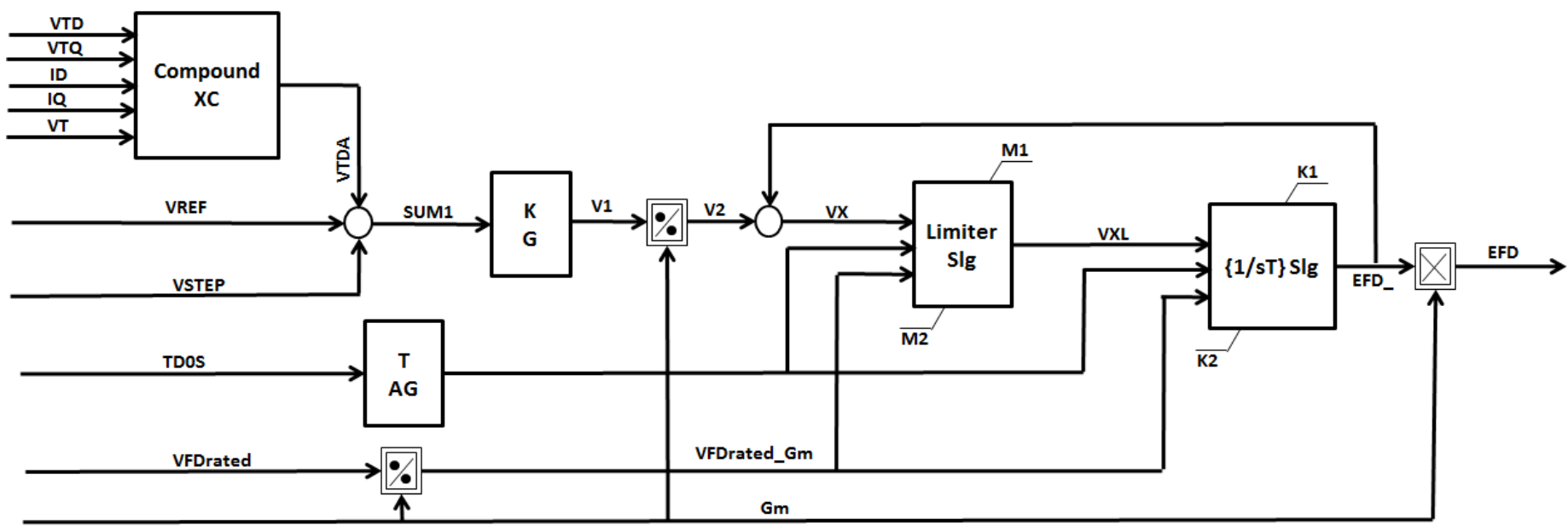

Fig. 3. The AVR Model in DIgSILENT

\section{B. Modelling of Component}

The important part for modelling the system is accomplishment the same result for active and reactive power flow from the generators in the PSCAD/EMTDC and DIgSILENT. In order to achieve the same P and Q for both systems, at first step, each generator in PSCAD/EMTDC has been modelled as a voltage source with a defined voltage angle and magnitude to obtain the specific P and Q. At the next step the voltage source has been replaced by generator and exactly the same voltage angle and magnitude has been used for the generator in order to have an exact $\mathrm{P}$ and $\mathrm{Q}$.

The real and reactive power are decided by three factors: the generator terminal voltage (magnitude and angle), the source voltage (magnitude and angle) and the impedance between generator and source.

In PSCAD/EMTDC, the generator starts as a source at $\mathrm{T}=0 \mathrm{~s}$. After a period of time, the exciter is put into operation. Then after a time period, which has to be set by user in "lock rotor-normal mode transition" part of "Variable Initialization Data" window, the governor is put into operation. Finally, the generator is put into operation. This performance of PSCAD/EMTDC makes possible for users to model the generator as a voltage source to obtain the voltage magnitude and angle.

During the transition from source to generator, the initial voltage and angle is important for a smooth transition. If the initial condition is far from the required final state, it takes a long time to reach to the steady state. Usually the initial conditions can be obtained from power flow programs (such as PSSE or DIgSILEN), or for a very simple case it can be calculated manually. In this case, the initial conditions are obtained from load flow solution from DIgSILENT (P and Q for each generator).

In PSCAD/EMTDC, there are two ways to let the generator output real power as required final result:

1- Selecting "control source P out" as "No" in "configuration-advanced" window. Then the generator will finally output the power specified in "initializing real power" of "variable initialization data".
2- Selecting "control source $\mathrm{P}$ out" as "Yes" in "configuration-advanced" window. Then the generator will finally output voltage magnitude and angle specified in "initial conditions". The real and reactive power is thereby decided by the voltage magnitude and angle.

In this study the second method has been applied.

As a last step, since the transformer which has been used for this system, is D-Y connection, there is 30 degree difference for voltage angle, which has to take into account for the initial voltage angle.

The used algorithm for this method can be summarized as Fig.2.

- Synchronous Generator

In power system dynamic studies, the synchronous generator is generally represented using the dq-axis. The sixth order model has been found to sufficiently represent the synchronous generator in stability studies and the equivalent circuit, which has been used to represent this model, has six electrical circuits: stator $\mathrm{d}$ and q-axis circuits, the field circuit, one d-axis damper winding and two q-axis damper windings.

The fifth order generator model is used to represent salient pole machines; this model is similar to the sixth order model, but has only one damper winding on the q-axis. These two models are available in both DIgSILENT and PSCAD software and both software tools also have fifth and sixth order degrees of complexity models [8].

In PSCAD/EMTDC, the generator can be represented by the infinite source series with a subtransient impedance matrix. The subtransient matrix contains $3 \times 3$ sub-matrixes of the form:

$\left[\begin{array}{ccc}X_{s} & X_{m} & X_{m} \\ X_{m} & X_{s} & X_{m} \\ X_{m} & X_{m} & X_{s}\end{array}\right]$

Where $X_{s}$ is the self-reactance of each phase and $X_{m}$ is the mutual reactance among the three phases. As in any other three-phase network component, these self and mutual 


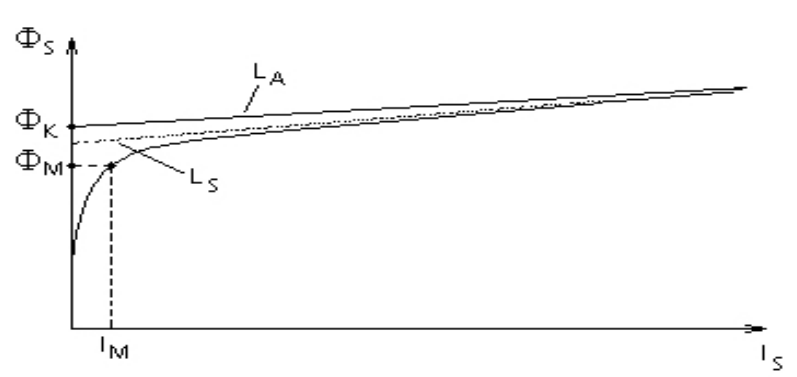

Fig. 4. Core Saturation Characteristic of the Classical Transformer[9]

reactances are related to the positive and zero sequence values, $\mathrm{X}_{1}$ and $\mathrm{X}_{0}$, by [9]:

$$
\begin{aligned}
& X_{s}=\frac{\left(X_{0}+2 X_{1}\right)}{3} \\
& X_{m}=\frac{\left(X_{0}-X_{1}\right)}{3}
\end{aligned}
$$

\section{- Generator Magnetic Saturation}

In DIgSILENT, the user sets two saturation parameters, $S_{1.0}$ and $S_{1.2}$, which are equivalent to 1.0 and 1.2 pu terminal voltage (flux linkage), respectively [8].

The open circuit saturation curve of the generator gives these parameters and they are computed using (4) - (6) as given below [8]. It should be mentioned that these equations do not apply if the generator is fully saturated.

$$
\begin{aligned}
& \psi_{I}=A_{s a t} e^{B_{r o t}\left(\psi_{a t}-\psi_{a t T 1}\right)} \\
& S_{1.0}=\frac{I_{A 1.0}-I_{B 1.0}}{I_{B 1.0}} \\
& S_{1.2}=\frac{I_{A 1.2}-I_{B 1.0}}{I_{B 1.2}}
\end{aligned}
$$

\section{Where:}

$\Psi_{a t}$ : Flux linkage at the point on the non-linear curve

$\Psi_{I}$ : Flux linkage drop due to saturation

$\Psi_{I}$ : Linear characteristic threshold flux linkage

$A_{\text {sat }}, B_{\text {sat }}:$ constants

$I_{A 1.0} I_{B 1.0} I_{A 1.2} I_{B 1.2}$ : Field currents

However, in PSCAD the user provides data for up to ten points on the non-linear open circuit saturation curve. The first point must be $(0,0)$ and the second point must lie on the linear part of the curve. The other points on the non-linear part of the curve are determined using (4) [8].

\section{- Excitation}

Static excitation systems supply direct current to the generator field winding through the rectifiers, which are fed by either transformers or auxiliary machine windings. A simplified version of this consists of voltage transducer delay, exciter and Transient Gain Reduction (TGR). The signal EPSS is a stabilising signal from the PSS, if one is used in conjunction with the exciter.

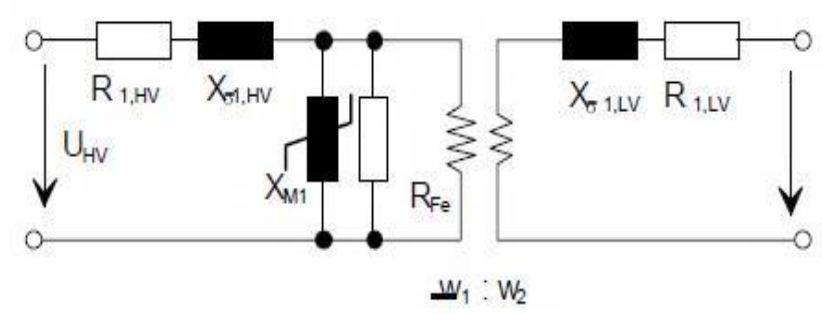

Fig.5. Equivalent Circuit of the 2 Winding 3-Phase for Positive Sequence[10]

The exciter model used in PSCAD/EMTDC is an IEEE type STlA excitation model and the schematic in PowerFactory that defines the AVR model is presented in Fig.3.

\section{- Transmission}

Throughout the work presented within this paper, in both PSCAD/EMTDC and DIgSILENT PowerFactory, transmission lines are modelled using a lumped parameter model and the common representation.

A simple $\mathrm{Pi}$ section model will give the correct fundamental impedance, but cannot accurately represent other frequencies unless many sections are used (which is inefficient). It also cannot accurately represent the frequency dependent parameters of a line (such as skin effect) [9].

\section{- $\quad$ Transformer}

Many transformer studies, however, do require core saturation to be adequately modelled. Saturation can be represented in one of two ways: First, with a varying inductance across the winding wound closest to the core or, second, with a compensating current source across the winding wound closest to the core.

In EMTDC, the current source representation is used, since it does not involve change to the subsystem matrix during saturation. For a two winding, single-phase transformer, saturation using a current source is shown in Fig.4.

The current $I_{S}(t)$ is a function of winding voltage $V_{L}(t)$. Winding flux $\Phi_{S}(t)$ is defined by assuming that the current $\mathrm{I}_{\mathrm{S}}(\mathrm{t})$ is the current in the equivalent non-linear saturating inductance $\mathrm{L}_{\mathrm{S}}(\mathrm{t})$. The air core inductance $\mathrm{L}_{\mathrm{A}}$ is represented by the straight-line characteristic, which bisects the flux axis at $\phi_{\mathrm{K}}$. The actual saturation characteristic is represented by $\mathrm{L}_{\mathrm{M}}$ and is asymptotic to both the vertical flux axis and the air core inductance characteristic. The sharpness of the knee point is defined by $\phi_{M}$ and $I_{M}$, which can represent the peak magnetizing flux and current at rated volts. It is possible to define an asymptotic equation for current in the non-linear saturating inductance $\mathrm{L}_{\mathrm{S}}$ if $\mathrm{L}_{\mathrm{A}}, \phi_{\mathrm{K}}, \phi_{\mathrm{M}}$, and $\mathrm{I}_{\mathrm{M}}$ are known.

This method is an approximate way of adding saturation to mutually coupled windings; however, it suffers from the disadvantage that, in most practical situations, the data is not available to make use of them; the saturation curve is rarely 

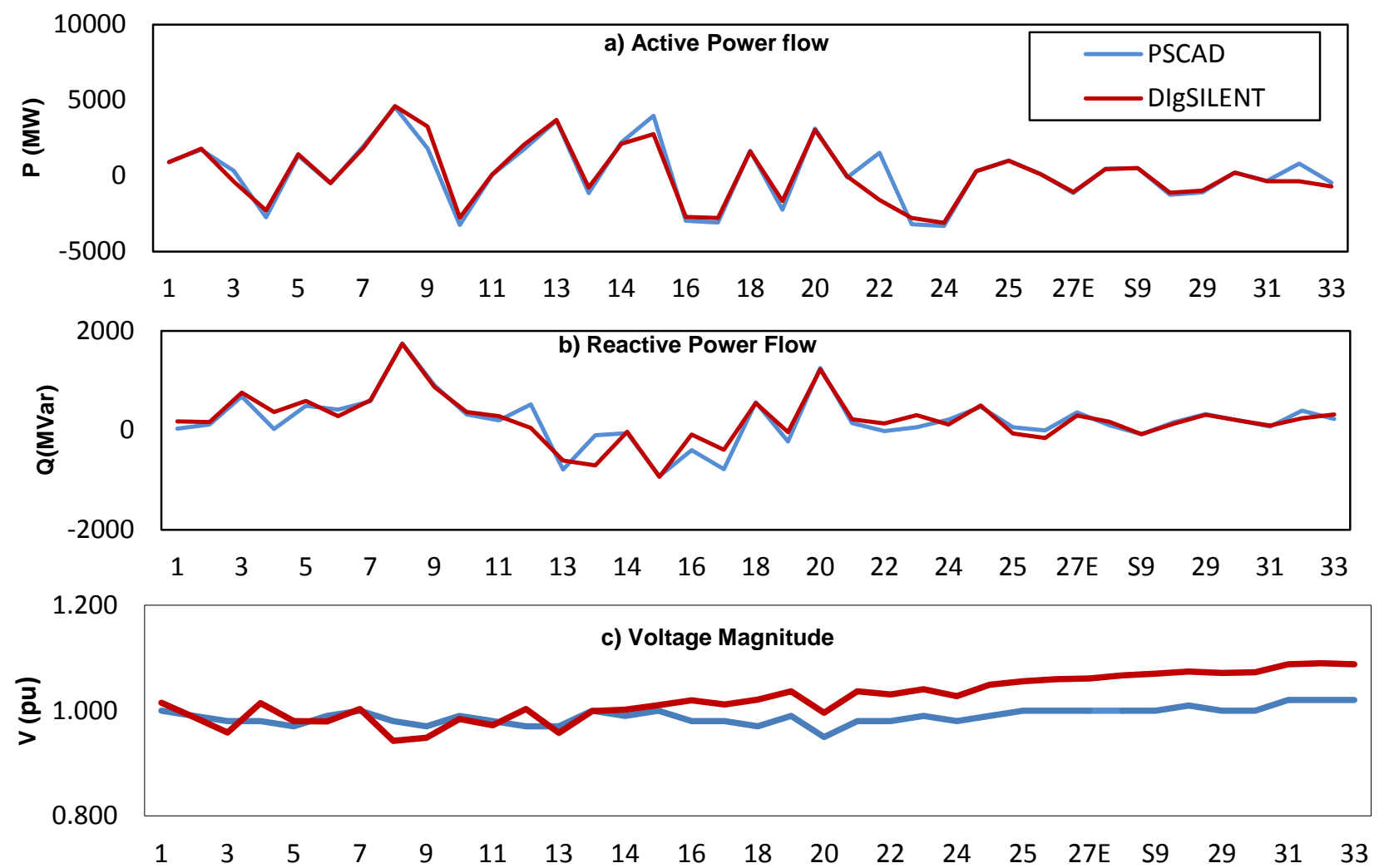

Fig. 6. The Power Flow Results and Voltage Magnitude in PSCAD/EMTDC and DIgSILENT

known much beyond the knee. The core and winding dimensions, and other related details, cannot be easily found.

Fig.5 shows the equivalent model of 2 winding 3-phase transformer for the positive sequence IN DIgSILENT. For simplicity, the tap changer has been omitted from the figure [10]. To have the same system in both software tools, the automatic tab changing has for the transformer been removed from the DIgSILENT. But aforementioned software tools have different saturation characteristic and as a result different data (such as distribution of leakage reactance and resistances, magnetizing impedance etc.) have to be define for the winding transformer component

\section{SiMULATION RESULT}

Both programs have graphical user interface. In the graphics environment, the user can draw the system as a oneline diagram (or three phase in PSCAD) and populate the system with data using the pop-up windows. The one distinct advantage of the graphical user interface is that the user is able to quickly modify the network topology and input data.

In PSCAD, the user has online access to input variables, i.e. the user can change parameters during a simulation. Switches, push buttons, sliders and ammeters are examples of the control and meter interfaces available in the program.

In DIgSILENT, the user is able to run a load flow before the dynamic simulation. PSCAD does not perform load flow calculations and, hence, the user should have pre-calculated initial conditions for all elements in the power system and this is weakness of using this software for modelling of the large system. The user starts a simulation without a disturbance and brings the system to steady state operation, after which the disturbance can be applied. [8]

The reduced model of the GB transmission system has been used for this paper, and is presented in Fig.1. The data has been defined, as much as possible, identically for the two software tools. But for some component such as generators and transformers, because of different characteristic, different information was needed.

\section{A. Steady-State Characteristic}

To compare the modelled system in PSCAD/EMTDC and DIgSILENT, the models must be verified. The active, reactive power and voltage magnitude for each zone in PSCAD/EMTC and DIgSILENT, are shown in Fig.6.

As can be seen, the results for active and reactive power flow for some zones are very close together, and for some other zones the difference is up to $20 \%$; this is because of different saturation characteristics of the transformer and the generator that was expected (Fig. 6.a and 6.b). In terms of voltage magnitude, the system follows exactly the same pattern for increasing and decreasing for each zone for both software tools, as shown in Fig.6.c. The larger difference is for Zone number 32, which is $5 \%$ difference.

\section{B. Dynamic Characteristic}

To investigate and compare the transient stability, the three phase fault has been applied at Zone 1 and the 


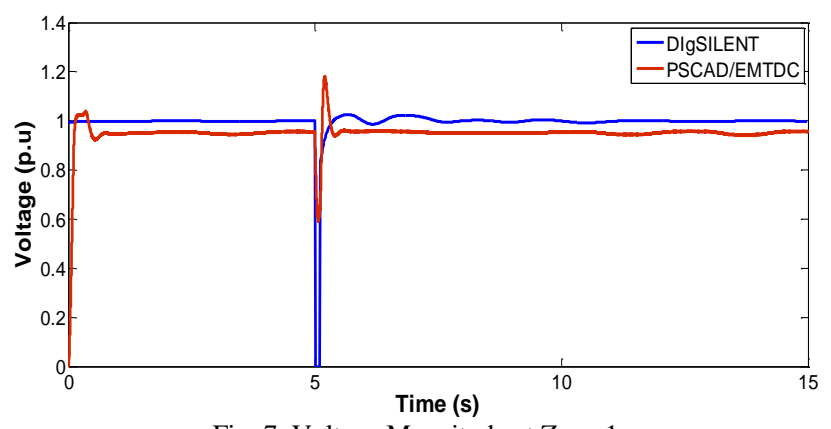

Fig. 7. Voltage Magnitude at Zone 1

behaviour of the G2 (nuclear generator) at this busbar, in terms of voltage and active power flow, has been monitored as shown in Fig.7 and Fig.8.

The pre-fault system condition base is:

DIgSILENT : $\mathrm{P}=1026 \mathrm{MW}$ and $\mathrm{V}=0.998$ p.u.

PSCAD/EMTDC : $\mathrm{P}=1056 \mathrm{MW}$ and $\mathrm{V}=0.96$ p.u.

The fault is cleared after $50 \mathrm{~ms}$, which is the critical fault clearing time for this model in PSCAD/EMTDC.

The aim of this study was to assess two aforementioned software tools. It should be mentioned that some of the data required to reproduce the case was not available, the modelling and data of AVR, governor and saturation curve for transformer and generator were not given, matching the absolute values of the results was difficult.

The simulation results for voltage magnitude for Zone 1 using the two tools are shown in Fig.8. It can be observed that the results obtained using DIgSILENT and PSCAD are similar, but, in post-fault, the PSACD results exhibited better damping than the other tool. In terms of voltage stability, both software tools agreed well when both the AVR and PSS were modelled. This may be attributed to the presence of the PSS, which damps the oscillations.

Fig.9 shows the result for active power flow in DIgSILENT and PSCAD. It can be seen that, for both software tools, the active power flow is not quite settled. The period of this oscillation in PSCAD in more than DIgSILENT, but the overshot in DIgSILENT is bigger than the PSCAD. This can be associated with the action of a governor, which improves the recovery of the power flow after disturbance. This system for stability analysis study needs further improvement and a different type of governor should be tested for this model.

\section{CONCLUSIONS}

In this paper, a reduced model of the GB transmission system was developed within a PSACD/EMTDC platform. The performance of the developed system was compared and confirmed with the DIgSILENT model, which was developed by National Grid. The results show that both models respond similarly to three phase to ground fault, although there are slight differences in the transient period and post-fault, which

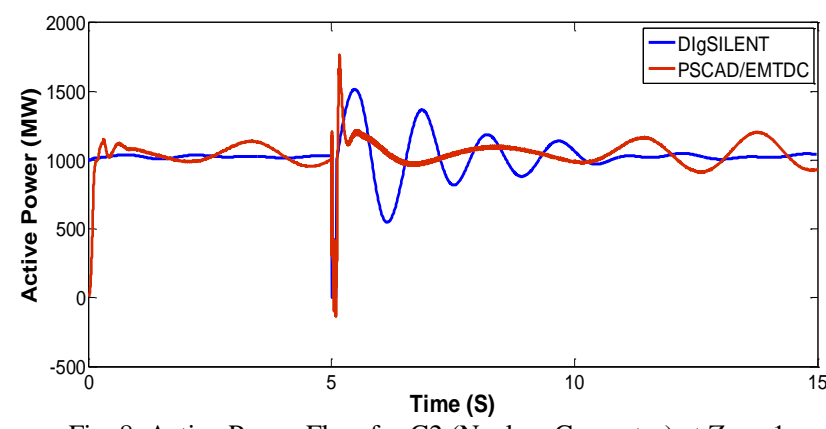

Fig. 8. Active Power Flow for G2 (Nuclear Generator) at Zone 1

might be due to numerical solving issues in the control system that are related to the different solvers used in the two software tools. Nevertheless, the results show, in terms of active and reactive power flow, that both software tools provide us with similar results.

The difference between the active and reactive power flow for some zones was expected, because the tools employ different models, components and analytical and numerical algorithms. Therefore, this model could be the power flow model, but, for stability analysis, it requires further improvement.

\section{REFERENCES}

[1] RenewableUK - UK Wind Energy Database (UKWED), [Online] Available at: www.bwea.com (accessed on:Feb 2014)

[2] L.P.Kunjumuhammed, B.C.Pal, N.F.Thornhill, . "A test system model for stability studies of UK power grid" PowerTech (POWERTECH), IEEE Grenoble, 2013, pp.1 - 6

[3] Energy \& Infrastructure Outlook offshore wind, The Crown Estate, 2013

[4] National Grid, "Electricity Ten Year Statement 2013"

[5] PSCAD, VersionV45 [Online]. Available at: https://hvdc.ca/pscad

[6] H.Teng ; C.Liu ; M.Han ; S.Ma ; X.Guo, 'IEEE9 Buses System Simulation and Modeling in PSCAD' Power and Energy Engineering Conference (APPEEC), 2010 Asia-Pacific

[7] O.K.P.Mokoka,; K.O. Awodele , 'Reliability Evaluation of Distribution Networks Using NEPLAN \& DIgSILENT Power Factory' AFRICON, 2013

[8] K.K.Kaberere .K.A Folly,A.I.Petroianu, ”Assessment of Commercially Available Software Tools for Transient Stability: Experience Gained in an Academic Environment". AFRICON, 2004. $7^{\text {th }}$ AFRICON Conference in Africa Volume:2, pp.711 - 716

[9] Chapter 3, Modelling of Power System in PSCAD/EMTDC, [online], Available Programhttp://archive.lib.cmu.ac.th/full/T/2009/enel0109tk_ch3.pdf

[10] PowerFactory,Version14.0.522.[Online].Available www.digsilent.com 